Eur. J. Math. Appl. (2021)1:12

URL: http://ejma.euap.org

(C) 2021 European Journal of Mathematics and Applications

\title{
INVERSE SUM INDEG INVARIANT OF SOME GRAPHS
}

\author{
S. NAGARAJAN ${ }^{1}$, P. MAHESH KUMAR ${ }^{1}$, AND K. PATTABIRAMAN $^{2, *}$
}

\begin{abstract}
The inverse sum indeg invariant $I S I(\Omega)$ of a simple graph $\Omega$ is defined as the sum of the terms $\frac{\gamma_{\Omega}(u) \gamma_{\Omega}(v)}{\gamma_{\Omega}(u)+\gamma_{\Omega}(v)}$ over all edges $u v$ of $\Omega$, where $\gamma_{\Omega}(u)$ denotes the degree of a vertex $u$ of $\Omega$. In this paper, we present several lower and upper bounds for inverse sum indeg invariant of some standard graphs.
\end{abstract}

\section{INTRODUCTION}

Molecular descriptors, results of functions mapping molecule's chemical information into a number [24], have found applications in modeling many physicochemical properties in QSAR and QSPR studies [3,11]. A particularly common type of molecular descriptors are those that are defined as functions of the structure of the underlying molecular graph, such as the Wiener invariabt [26], the Zagreb indices [9], the Randić invariabt [19] or the Balaban J-invariabt [1]. Damir Vukicević and Marija Gasperov [25] observed that many of these descritors are defined simply as the sum of individual bond contributions.

Among the 148 discrete Adriatic indices studied in [25], whose predictive properties were evaluated against the benchmark datasets of the Internation Academy of Mathematical Chemistry [16], 20 invariants were selected as significant predictors of physicochemical properties. In this connection, Sedlar et al. [20] studied the properties of the inverse sum indeg invariant, the descriptor that was selected in [25] as a significant predictor of total surface area of octane isomers and for which the extremal graphs obtained with the help of Math. Chem. have a particularly simple and elegant structure. The inverse sum indeg invariant is defined as

$I S I(\Omega)=\sum_{u v \in E(\Omega)} \frac{1}{\frac{1}{\gamma_{\Omega^{(u)}}}+\frac{1}{\gamma_{\Omega}(v)}}=\sum_{u v \in E(\Omega)} \frac{\gamma_{\Omega}(u) \gamma_{\Omega}(v)}{\gamma_{\Omega}(u)+\gamma_{\Omega}(v)}$.

Extremal values of inverse sum indeg invariant across several graph classes, including connected graphs, chemical graphs, trees and chemical trees were determined in [20]. The bounds of a descriptor are important information of a molecular graph in the sense that they establish the approximate range of the descriptor in terms of molecular structural parameters. In [6], some sharp bounds for the inverse sum indeg invariant of connected graphs are given. The inverse sum indeg invariant of some nanotubes is computed in [7]. In this connection, we present

\footnotetext{
${ }^{1}$ Department of Mathematics, Kongu Arts and Science College(Autonomous), Erode-638107, INDIA

${ }^{2}$ Department of Mathematics, Government Arts College(Autonomous), Kumbakonam-612 002 , INDIA

${ }^{*}$ Corresponding AUTHOR

E-mail addresses: profnagarajan.s@gmail.com, mathsmaheshkumar@gmail.com, pramank@gmail.com.

Key words and phrases. degree; inverse sum indeg invariant; derived graph.

Received 04/09/2021.
} 
several upper and lower bounds on the inverse sum indeg invariant in terms of some molecular structural parameters and relate this invariant to various well-known molecular descriptors.

The Zagreb indices are amoung the oldest topological indices, and were introduced by Gutman and Trinajstić [9] in 1972. These indices have since been used to study molecular complexity, chirality, ZE-ismerism and hetero-systems. The first and second Zagreb indices of $\Omega$ are denoted by $M_{1}(\Omega)$ and $M_{2}(\Omega)$, respectively, and defined as $M_{1}(\Omega)=\sum_{v \in V(\Omega)}\left(\gamma_{\Omega}(v)\right)^{2}=$ $\sum_{u v \in E(\Omega)}\left(\gamma_{\Omega}(u)+\gamma_{\Omega}(v)\right)$ and $M_{2}(\Omega)=\sum_{u v \in E(\Omega)} \gamma_{\Omega}(u) \gamma_{\Omega}(v)$

Another variant of the Randić connectivity invariant named the harmonic invariant was introduced by Fajtlowicz [4] in 1987. The harmonic invariant $H(\Omega)$ of $\Omega$ is defined as $H(\Omega)=$

$\sum_{u v \in E(\Omega)} \frac{1}{\gamma_{\Omega}(u)+\gamma_{\Omega}(v)}$. The inverse degree of a connected graph $\Omega$ is defined as $I D(\Omega)=\sum_{u \in E(\Omega)} \frac{1}{\gamma_{\Omega}(u)}$. In this paper, we present several lower and upper bounds for inverse sum indeg invariant of some derived graphs

\section{LOWER BOUNDS FOR ISI}

In this section, we obtain the lower bounds for inverse sum indeg invariant of a connected graph.

Theorem 2.1. Let $\Omega$ be a graph with $m$ edges. Then $I S I(\Omega) \geq m\left(1+\ln \left(\frac{\delta(\Omega)^{2}}{2 \Delta(\Omega)}\right)\right)$ with equality if and only if $\Omega$ is 2-regular graph.

Proof. Assume that the function $f(x)=x-\ln x-1$. One can easily prove that $f(x) \geq 0$.

Hence for any edge $u v \in E(\Omega)$,

$$
\frac{\gamma_{\Omega}(u) \gamma_{\Omega}(v)}{\gamma_{\Omega}(u)+\gamma_{\Omega}(v)}-\ln \left(\frac{\gamma_{\Omega}(u) \gamma_{\Omega}(v)}{\gamma_{\Omega}(u)+\gamma_{\Omega}(v)}\right)-1 \geq 0 .
$$

So,

$$
\frac{\gamma_{\Omega}(u) \gamma_{\Omega}(v)}{\gamma_{\Omega}(u)+\gamma_{\Omega}(v)} \geq 1+\ln \left(\frac{\gamma_{\Omega}(u) \gamma_{\Omega}(v)}{\gamma_{\Omega}(u)+\gamma_{\Omega}(v)}\right)
$$

By taking the summation over the edges of the graph, we get

$$
\begin{aligned}
I S I(\Omega) & \geq m+\sum_{u v \in E(\Omega)} \ln \left(\frac{\gamma_{\Omega}(u) \gamma_{\Omega}(v)}{\gamma_{\Omega}(u)+\gamma_{\Omega}(v)}\right) \\
& =m+\ln \left(\prod_{u v \in E(\Omega)} \frac{\gamma_{\Omega}(u) \gamma_{\Omega}(v)}{\gamma_{\Omega}(u)+\gamma_{\Omega}(v)}\right) \\
& =m+\ln \left(\prod_{u v \in E(\Omega)} \frac{\delta(\Omega)^{2}}{2 \Delta(\Omega)}\right) \\
& =m+\ln \left(\frac{\delta(\Omega)^{2}}{2 \Delta(\Omega)}\right)^{m} \\
& =m+m \ln \left(\frac{\delta(\Omega)^{2}}{2 \Delta(\Omega)}\right) .
\end{aligned}
$$

Hence

$$
I S I(\Omega) \geq m\left(1+\ln \left(\frac{\delta(\Omega)^{2}}{2 \Delta(\Omega)}\right)\right) .
$$


To show the equality, let $f(x)=0$, then $x=1$. So, $\frac{\gamma_{\Omega}(u) \gamma_{\Omega}(v)}{\gamma_{\Omega}(u)+\gamma_{\Omega}(v)}=1$, for any edge $u v \in E(\Omega)$. Hence $\gamma_{\Omega}(u) \gamma_{\Omega}(v)=\gamma_{\Omega}(u)+\gamma_{\Omega}(v)$, which holds if and only if $\Omega$ is 2-regular graph.

Theorem 2.2. If $\Omega$ is a graph with $n$ vertices and $m$ edges. Then $I S I(\Omega) \geq 2 m-n$ with equality if and only if $\Omega$ is 2-regular graph.

Proof. Assume that the function $f(x)=x+\frac{1}{x}-2$. One can easily prove that $f(x) \geq 0$.

Hence for any edge $u v \in E(\Omega)$,

$$
\frac{\gamma_{\Omega}(u) \gamma_{\Omega}(v)}{\gamma_{\Omega}(u)+\gamma_{\Omega}(v)}+\frac{\gamma_{\Omega}(u)+\gamma_{\Omega}(v)}{\gamma_{\Omega}(u) \gamma_{\Omega}(v)}-2 \geq 0
$$

By taking the summation over the edges of the graph, we get

$$
I S I(\Omega)+n-2 m \geq 0 .
$$

Hence

$$
I S I(\Omega) \geq 2 m-n \text {. }
$$

To show the equality, let $f(x)=0$, then $x=1$. The rest of the proof is similar to that in Theorem 2.1.

\section{VERTEX- EDGE CORONA PRODUCT GRAPH}

Let $\Omega_{1}$ be a graph with vertex set $V\left(\Omega_{1}\right)=\left\{x_{1}, x_{2}, \ldots, x_{n_{1}}\right\}$ and edge set $E\left(\Omega_{1}\right)=$ $\left\{e_{1}, e_{2}, \ldots, e_{m_{1}}\right\}$. Let $\Omega_{2}$ be a graph with vertex set $V\left(\Omega_{2}\right)=\left\{y_{1}, y_{2}, \ldots, y_{n_{2}}\right\}$ and edge set $E\left(\Omega_{1}\right)=\left\{e_{1}^{\prime}, e_{2}^{\prime}, \ldots, e_{m_{2}}^{\prime}\right\}$. The vertex-edge corona of $\Omega_{1}$ and $\Omega_{2}$, denoted by $\Omega_{1} \bullet \Omega_{2}$, is the graph obtained by taking one copy of $\Omega_{1},\left|V\left(\Omega_{1}\right)\right|$ copies of $\Omega_{2}$ and $\left|E\left(\Omega_{1}\right)\right|$ copies of $\Omega_{2}$, then joining $i^{t h}$ vertex of $\Omega_{1}$ to every vertex in the $i^{\text {th }}$ vertex copy of $\Omega_{2}$ and also joining end vertices of $j^{\text {th }}$ edge of $\Omega_{1}$ to every vertex in $j^{\text {th }}$ edge copy of $\Omega_{2}$, where $1 \leq i \leq n_{1}$ and $1 \leq j \leq m_{1}$. One can see that $\left|V\left(\Omega_{1} \bullet \Omega_{2}\right)\right|=n_{1}+n_{2}\left(m_{1}+n_{1}\right)$ and $\left|E\left(\Omega_{1} \bullet \Omega_{2}\right)\right|=m_{1}+m_{1}\left(m_{2}+2 n_{2}\right)+n_{1}\left(m_{2}+n_{2}\right)$. We denote the vertex set of the $j^{\text {th }}$ edge copy of $\Omega_{2}$ by $V_{j e}\left(\Omega_{2}\right)=\left\{x_{j 1}, x_{j 2}, \ldots, x_{j n_{2}}\right\}$ and the vertes set of the $i^{t h}$ vertex copy of $\Omega_{2}$ by $V_{i v}\left(\Omega_{2}\right)=\left\{w_{i 1}, w_{i 2}, \ldots, w_{i n_{2}}\right\}$. Also, we denote by $E_{j e}\left(\Omega_{2}\right)$ and $E_{i v}\left(\Omega_{2}\right)$, the set of the $j^{t h}$ edge $i^{t h}$ vertex copy of $\Omega_{2}$, respectively.

Lemma 3.1. [28] Let $f$ be a convex function on the interval $I$ and $x_{1}, x_{2}, \ldots, x_{n} \in I$. Then $f\left(\frac{x_{1}+x_{2}+\ldots+x_{n}}{n}\right) \leq \frac{f\left(x_{1}\right)+f\left(x_{2}\right)+\ldots f\left(x_{n}\right)}{n}$, with equality if and only if $x_{1}=x_{2}=\ldots=x_{n}$.

Theorem 3.2. Let $G_{i}$ be $\left(n_{i}, m_{i}\right)$ graph, $i=\{1,2\}$. Then $I S I\left(\Omega_{1} \bullet \Omega_{2}\right) \leq\left(\frac{n_{2}+1}{4}\right) I S I\left(\Omega_{1}\right)+$ $\left(\frac{n_{1}+m_{1}}{4}\right) I S I\left(\Omega_{2}\right)+\left(\frac{n_{2}\left(4 m_{2}+5 n_{2}\right)}{8\left(n_{2}+1\right)}\right) H\left(\Omega_{1}\right)+\left(\frac{4 m_{1}+n_{1}}{8}\right) H\left(\Omega_{2}\right)+\left(\frac{\left(n_{2}+1\right)\left(2 I D\left(\Omega_{2}\right)+3 n_{2}+2 m_{2}+2\right)}{16}\right) M_{1}\left(\Omega_{1}\right)+$ $\left(\frac{n_{1}+m_{1}}{8}\right) M_{1}\left(\Omega_{2}\right)+\left(\frac{\left(n_{2}+1\right)^{2}}{8 n_{2}}\right) M_{2}\left(\Omega_{1}\right)+\left(\frac{2 n_{1}+m_{1}}{16}\right) M_{2}\left(\Omega_{2}\right)+\left(\frac{2 n_{2} m_{2}+n_{2}^{2}}{16\left(n_{2}+1\right)}\right) I D\left(\Omega_{1}\right)+$ $\left(\frac{n_{1} n_{2}+3 n_{2} m_{1}+2 m_{1}}{4}\right) I D\left(\Omega_{2}\right)+\frac{1}{16}\left(2 n_{2} m_{1}\left(3 n_{2}+6 m_{2}+8\right)+2 n_{1}\left(m_{1}+m_{2}\right)+n_{1} n_{2}\left(2 n_{2}+\right.\right.$ 1) $\left.+32 m_{1} m_{2}\right)+\left(\frac{n_{1} n_{2}\left(2 m_{2}+n_{2}\right)}{16\left(n_{2}+1\right)}\right)$.

Proof. By using the definition of $I S I$ of $\Omega_{1} \bullet \Omega_{2}$, we have

$$
\begin{aligned}
& I S I\left(\Omega_{1} \bullet \Omega_{2}\right)=\sum_{x y \in E\left(\Omega_{1} \bullet \Omega_{2}\right)} \frac{\gamma_{\Omega_{1} \bullet \Omega_{2}}(x) \gamma_{\Omega_{1} \bullet \Omega_{2}}(y)}{\gamma_{\Omega_{1} \bullet \Omega_{2}}(x)+\gamma_{\Omega_{1} \bullet \Omega_{2}}(y)} \\
& =I_{1}+I_{2}+I_{3}+I_{4}+I_{5} \text {, where }
\end{aligned}
$$




$$
\begin{aligned}
I_{1} & =\sum_{x_{i} x_{j} \in E\left(\Omega_{1}\right)} \frac{\gamma_{\Omega_{1} \bullet \Omega_{2}}\left(x_{i}\right) \gamma_{\Omega_{1}} \bullet \Omega_{2}\left(x_{j}\right)}{\gamma_{\Omega_{1}} \bullet \Omega_{2}\left(x_{i}\right)+\gamma_{\Omega_{1}} \bullet \Omega_{2}\left(x_{j}\right)}, \\
I_{2} & =\sum_{e_{i} \in E\left(\Omega_{1}\right)} \sum_{x_{i j} x_{i k} \in E_{i e}\left(\Omega_{2}\right)} \frac{\gamma_{\Omega_{1} \bullet \Omega_{2}}\left(x_{i j}\right) \gamma_{\Omega_{1}} \bullet \Omega_{2}\left(x_{i k}\right)}{\gamma_{\Omega_{1}} \bullet \Omega_{2}\left(x_{i j}\right)+\gamma_{\Omega_{1}} \bullet \Omega_{2}\left(x_{i k}\right)}, \\
I_{3} & =\sum_{e_{i}=x_{l} x_{m} \in E\left(\Omega_{1}\right)} \sum_{x_{i j} \in V_{i e}\left(\Omega_{2}\right)} \frac{\left(\gamma_{\Omega_{1} \bullet \Omega_{2}}\left(x_{l}\right)+\gamma_{\Omega_{1}} \bullet \Omega_{2}\left(x_{m}\right)\right) \gamma_{\Omega_{1} \bullet \Omega_{2}}\left(x_{i j}\right)}{\gamma_{\Omega_{1} \bullet \Omega_{2}}\left(x_{l}\right)+\gamma_{\Omega_{1}} \bullet \Omega_{2}\left(x_{m}\right)+\gamma_{\Omega_{1}} \bullet \Omega_{2}\left(x_{i j}\right)} \\
I_{4} & =\sum_{x_{i} \in V\left(\Omega_{1}\right)} \sum_{w_{i j} w_{i k} \in E_{i v}\left(\Omega_{2}\right)} \frac{\gamma\left(w_{i j}\right) \gamma\left(w_{i k}\right)}{\gamma\left(w_{i j}\right)+\gamma\left(w_{i k}\right)} \text { and } \\
I_{5} & =\sum_{x_{i} \in V\left(\Omega_{1}\right)} \sum_{w_{i j} \in V_{i v}\left(\Omega_{2}\right)} \frac{\gamma\left(x_{i}\right) \gamma\left(w_{i j}\right)}{\gamma\left(x_{i}\right)+\gamma\left(w_{i j}\right)} .
\end{aligned}
$$

From the structure of the graph $\Omega_{1} \bullet \Omega_{2}$, we have the following;

。 If $x_{i} \in V\left(\Omega_{1}\right)$, then $\gamma_{\Omega_{1} \bullet \Omega_{2}}\left(x_{i}\right)=\left(n_{2}+1\right) \gamma_{\Omega_{1}}\left(x_{i}\right)+n_{2}$.

o If $x_{i j} \in V_{i v}\left(\Omega_{2}\right)$, then $\gamma_{\Omega_{1} \bullet \Omega_{2}}\left(x_{i j}\right)=\gamma_{\Omega_{2}}\left(y_{j}\right)+2$.

○ If $w_{i j} \in V_{i v}\left(\Omega_{2}\right)$, then $\gamma_{\Omega_{1} \bullet \Omega_{2}}\left(w_{i j}\right)=\gamma_{\Omega_{2}}\left(w_{j}\right)+1$. Hence

$$
\begin{aligned}
I_{1} & =\sum_{x_{i} x_{j} \in E\left(\Omega_{1}\right)} \frac{\gamma_{\Omega_{1} \bullet \Omega_{2}}\left(x_{i}\right) \gamma_{\Omega_{1} \bullet \Omega_{2}}\left(x_{j}\right)}{\gamma_{\Omega_{1} \bullet \Omega_{2}}\left(x_{i}\right)+\gamma_{\Omega_{1}} \bullet \Omega_{2}\left(x_{j}\right)} \\
& =\sum_{x_{i} x_{j} \in E\left(\Omega_{1}\right)} \frac{\left(\left(n_{2}+1\right) \gamma_{\Omega_{1}}\left(x_{i}\right)+n_{2}\right)\left(\left(n_{2}+1\right) \gamma_{\Omega_{1}}\left(x_{j}\right)+n_{2}\right)}{\left(n_{2}+1\right) \gamma_{\Omega_{1}}\left(x_{i}\right)+n_{2}+\left(n_{2}+1\right) \gamma_{\Omega_{1}}\left(x_{j}\right)+n_{2}} \\
& =\sum_{x_{i} x_{j} \in E\left(\Omega_{1}\right)} \frac{\left(n_{2}+1\right)^{2}\left(\gamma_{\Omega_{1}}\left(x_{i}\right) \gamma_{\Omega_{1}}\left(x_{j}\right)\right)+n_{2}^{2}+n_{2}\left(n_{2}+1\right)\left(\gamma_{\Omega_{1}}\left(x_{i}\right)+\gamma_{\Omega_{1}}\left(x_{j}\right)\right)}{\left(n_{2}+1\right)\left(\gamma_{\Omega_{1}}\left(x_{i}\right)+\gamma_{\Omega_{1}}\left(x_{j}\right)+2 n_{2}\right)} .
\end{aligned}
$$

Jensen's inequality is used for the convex function $f: \mathcal{R}_{+} \rightarrow \mathcal{R}_{+}, f(x)=\frac{1}{x}$ according to Lemma 3.1, we have $\frac{1}{\left(\gamma_{\Omega_{1}}\left(x_{i}\right)+\gamma_{\Omega_{1}}\left(x_{j}\right)+2 n_{2}\right)}=\frac{1}{4\left(\gamma_{\Omega_{1}}\left(x_{i}\right)+\gamma_{\Omega_{1}}\left(x_{j}\right)\right)}+\frac{1}{8 n_{2}}$ with equality if and only if $\gamma_{\Omega_{1}}\left(x_{i}\right)+\gamma_{\Omega_{1}}\left(x_{j}\right)=2 n_{2}$. Thus

$$
\begin{aligned}
& I_{1} \leq \sum_{x_{i} x_{j} \in E\left(\Omega_{1}\right)} \frac{\left(n_{2}+1\right)^{2}\left(\gamma_{\Omega_{1}}\left(x_{i}\right) \gamma_{\Omega_{1}}\left(x_{j}\right)\right)+n_{2}\left(n_{2}+1\right)\left(\gamma_{\Omega_{1}}\left(x_{i}\right)+\gamma_{\Omega_{1}}\left(x_{j}\right)+n_{2}^{2}\right)}{4\left(n_{2}+1\right)\left(\gamma_{\Omega_{1}}\left(x_{i}\right)+\gamma_{\Omega_{1}}\left(x_{j}\right)\right)} \\
& +\frac{\left(n_{2}+1\right)^{2}\left(\gamma_{\Omega_{1}}\left(x_{i}\right) \gamma_{\Omega_{1}}\left(x_{j}\right)\right)+n_{2}\left(n_{2}+1\right)\left(\gamma_{\Omega_{1}}\left(x_{i}\right)+\gamma_{\Omega_{1}}\left(x_{j}\right)+n_{2}^{2}\right)}{8 n_{2}} \\
& =\frac{1}{4} I S I\left(\Omega_{1}\right)\left(n_{2}+1\right)+\frac{n_{2} m_{1}}{4}+\frac{n_{2}^{2}}{8\left(n_{2}+1\right)} H\left(\Omega_{1}\right) \\
& +\frac{\left(n_{2}+1\right)^{2}}{8 n_{2}} M_{2}\left(\Omega_{1}\right)+\frac{\left(n_{2}+1\right)}{8} M_{1}\left(\Omega_{1}\right)+\frac{n_{2} m_{1}}{8} \\
& =\frac{\left(n_{2}+1\right)}{4} I S I\left(\Omega_{1}\right)+\frac{n_{2}^{2}}{8\left(n_{2}+1\right)} H\left(\Omega_{1}\right)+\frac{\left(n_{2}+1\right)^{2}}{8 n_{2}} M_{2}\left(\Omega_{1}\right) \\
& +\frac{\left(n_{2}+1\right)}{8} M_{1}\left(\Omega_{1}\right)+\frac{3 n_{2} m_{1}}{8} \text {. } \\
& I_{2}=\sum_{e_{i} \in E\left(\Omega_{1}\right)} \sum_{x_{i j} x_{i k} \in E_{i e}\left(\Omega_{2}\right)} \frac{\gamma_{\Omega_{1} \bullet \Omega_{2}}\left(x_{i j}\right) \gamma_{\Omega_{1} \bullet \Omega_{2}}\left(x_{i k}\right)}{\gamma_{\Omega_{1} \bullet \Omega_{2}}\left(x_{i j}\right)+\gamma_{\Omega_{1} \bullet \Omega_{2}}\left(x_{i k}\right)} \\
& =\sum_{e_{i} \in E\left(\Omega_{1}\right)} \sum_{y_{j} y_{k} \in E\left(\Omega_{2}\right)} \frac{\left(\gamma_{\Omega_{2}}\left(y_{j}\right)+2\right)\left(\gamma_{\Omega_{2}}\left(y_{k}\right)+2\right)}{\gamma_{\Omega_{2}}\left(y_{j}\right)+\gamma_{\Omega_{2}}\left(y_{k}\right)+4} .
\end{aligned}
$$


Apply Jensen's inequality, we have $\frac{1}{\gamma_{\Omega_{2}}\left(y_{j}\right)+\gamma_{\Omega_{2}}\left(y_{k}\right)+4} \leq \frac{1}{4\left(\gamma_{\Omega_{2}}\left(y_{j}\right)+\gamma_{\Omega_{2}}\left(y_{k}\right)\right)}+\frac{1}{16}$ with equality if and only if $\gamma_{\Omega_{2}}\left(y_{j}\right)+\gamma_{\Omega_{2}}\left(y_{k}\right)=4$. Thus

$$
\begin{aligned}
& I_{2} \leq \frac{1}{4} \sum_{e_{i} \in E\left(\Omega_{1}\right)} \sum_{y_{j} y_{k} \in E\left(\Omega_{2}\right)}\left(\frac{\left(\gamma_{\Omega_{2}}\left(y_{j}\right)+2\right)\left(\gamma_{\Omega_{2}}\left(y_{k}\right)+2\right)}{\gamma_{\Omega_{2}}\left(y_{j}\right)+\gamma_{\Omega_{2}}\left(y_{k}\right)}\right. \\
& \left.+\frac{\left(\gamma_{\Omega_{2}}\left(y_{j}\right)+2\right)\left(\gamma_{\Omega_{2}}\left(y_{k}\right)+2\right)}{4}\right) \\
& =\frac{1}{4} \sum_{e_{i} \in E\left(\Omega_{1}\right)} \sum_{y_{j} y_{k} \in E\left(\Omega_{2}\right)}\left(\frac{\gamma_{\Omega_{2}}\left(y_{j}\right) \gamma_{\Omega_{2}}\left(y_{k}\right)}{\gamma_{\Omega_{2}}\left(y_{j}\right)+\gamma_{\Omega_{2}}\left(y_{k}\right)}+2\left(\gamma_{\Omega_{2}}\left(y_{j}\right)+\gamma_{\Omega_{2}}\left(y_{k}\right)\right)\right. \\
& +\frac{4}{\gamma_{\Omega_{2}}\left(y_{j}\right) \gamma_{\Omega_{2}}\left(y_{k}\right)} \\
& \left.+\frac{\gamma_{\Omega_{2}}\left(y_{j}\right) \gamma_{\Omega_{2}}\left(y_{k}\right)+2\left(\gamma_{\Omega_{2}}\left(y_{j}\right)+\gamma_{\Omega_{2}}\left(y_{k}\right)\right)+4}{4}\right) \\
& =\frac{1}{4} \sum_{e_{i} \in E\left(\Omega_{1}\right)}\left(I S I\left(\Omega_{2}\right)+3 m_{2}+2 H\left(\Omega_{2}\right)+\frac{M_{2}\left(\Omega_{2}\right)}{4}+\frac{M_{1}\left(\Omega_{2}\right)}{2}\right) \\
& =\frac{m_{1}}{4}\left(I S I\left(\Omega_{2}\right)+2 H\left(\Omega_{2}\right)+\frac{M_{2}\left(\Omega_{2}\right)}{4}+\frac{M_{1}\left(\Omega_{2}\right)}{2}+3 m_{2}\right) . \\
& I_{3}=\sum_{e_{i}=x_{l} x_{m} \in E\left(\Omega_{1}\right)} \sum_{x_{i} \in V_{i e}\left(\Omega_{2}\right)} \frac{\left(\gamma_{\Omega_{1} \bullet \Omega_{2}}\left(x_{l}\right)+\gamma_{\Omega_{1} \bullet \Omega_{2}}\left(x_{m}\right)\right) \gamma_{\Omega_{1} \bullet \Omega_{2}}\left(x_{i j}\right)}{\gamma_{\Omega_{1} \bullet \Omega_{2}}\left(x_{l}\right)+\gamma_{\Omega_{1} \bullet \Omega_{2}}\left(x_{m}\right)+\gamma_{\Omega_{1} \bullet \Omega_{2}}\left(x_{i j}\right)} \\
& =\sum_{e_{i} \in E\left(\Omega_{1}\right)} \sum_{y_{j} \in V\left(\Omega_{2}\right)} \frac{\left(\left(n_{2}+1\right) \gamma_{\Omega_{1}}\left(x_{l}\right)+n_{2}+\left(n_{2}+1\right) \gamma_{\Omega_{1}}\left(x_{m}\right)+n_{2}\right)\left(\gamma_{\Omega_{2}}\left(y_{j}\right)+2\right)}{\left(\left(n_{2}+1\right) \gamma_{\Omega_{1}}\left(x_{l}\right)+n_{2}+\left(n_{2}+1\right) \gamma_{\Omega_{1}}\left(x_{m}\right)+n_{2}\right)+\gamma_{\Omega_{2}}\left(y_{j}\right)+2} \\
& =\sum_{e_{i} \in E\left(\Omega_{1}\right)} \sum_{y_{j} \in V\left(\Omega_{2}\right)} \frac{\left(\left(n_{2}+1\right)\left(\gamma_{\Omega_{1}}\left(x_{l}\right)+\gamma_{\Omega_{1}}\left(x_{m}\right)\right)+2 n_{2}\right)\left(\gamma_{\Omega_{2}}\left(y_{j}\right)+2\right)}{\left(n_{2}+1\right)\left(\gamma_{\Omega_{1}}\left(x_{l}\right)+\gamma_{\Omega_{1}}\left(x_{m}\right)\right)+\gamma_{\Omega_{2}}\left(y_{j}\right)+\left(2 n_{2}+2\right)} \\
& \leq \sum_{e_{i} \in E\left(\Omega_{1}\right)} \sum_{y_{j} \in V\left(\Omega_{2}\right)} \frac{1}{4}\left(\left(n_{2}+1\right)\left(\gamma_{\Omega_{1}}\left(x_{l}\right)+\gamma_{\Omega_{1}}\left(x_{m}\right)\right)+2 n_{2}\right)\left(\gamma_{\Omega_{2}}\left(y_{j}\right)+2\right) \\
& \left(\frac{1}{\left(n_{2}+1\right)\left(\gamma_{\Omega_{1}}\left(x_{l}\right)+\gamma_{\Omega_{1}}\left(x_{m}\right)\right)}+\frac{1}{\gamma_{\Omega_{2}}\left(y_{j}\right)+2 n_{2}+2}\right) \\
& =\frac{1}{4} \sum_{e_{i} \in E\left(\Omega_{1}\right)} \sum_{y_{j} \in V\left(\Omega_{2}\right)}\left(\left(\gamma_{\Omega_{2}}\left(y_{j}\right)+2\right)+\frac{2 n_{2}\left(\gamma_{\Omega_{2}}\left(y_{j}\right)+2\right)}{\left(n_{2}+1\right)\left(\gamma_{\Omega_{1}}\left(x_{l}\right)+\gamma_{\Omega_{1}}\left(x_{m}\right)\right)}\right) \\
& +\frac{\left(n_{2}+1\right)\left(\gamma_{\Omega_{1}}\left(x_{l}\right)+\gamma_{\Omega_{1}}\left(x_{m}\right)\right)+2 n_{2}\left(\gamma_{\Omega_{2}}\left(y_{j}\right)+2\right)}{\gamma_{\Omega_{2}}\left(y_{j}\right)+2 n_{2}+2} \\
& =I_{3}^{\prime}+I_{3}^{\prime \prime} \text {, where } \\
& I_{3}^{\prime}=\frac{1}{4} \sum_{e_{i} \in E\left(\Omega_{1}\right)} \sum_{y_{j} \in V\left(\Omega_{2}\right)}\left(\gamma_{\Omega_{2}}\left(y_{j}\right)+2+\frac{2 n_{2}\left(\gamma_{\Omega_{2}}\left(y_{j}\right)+2\right)}{\left(n_{2}+1\right)\left(\gamma_{\Omega_{1}}\left(x_{l}\right)+\gamma_{\Omega_{1}}\left(x_{m}\right)\right)}\right) \\
& =\frac{1}{4} \sum_{e_{i} \in E\left(\Omega_{1}\right)}\left(2 m_{2}+2 n_{2}+\frac{2 n_{2}\left(2 m_{2}+2 n_{2}\right)}{\left(n_{2}+1\right)\left(\gamma_{\Omega_{1}}\left(x_{l}\right)+\gamma_{\Omega_{1}}\left(x_{m}\right)\right)}\right) \\
& =\frac{1}{4}\left(\left(2 m_{2}+2 n_{2}\right) m_{1}+\frac{n_{2}\left(2 m_{2}+2 n_{2}\right)}{\left(n_{2}+1\right)} H\left(\Omega_{1}\right)\right) .
\end{aligned}
$$




$$
\begin{aligned}
& I_{3}^{\prime \prime}=\frac{1}{4} \sum_{e_{i} \in E\left(\Omega_{1}\right)} \sum_{y_{j} \in V\left(\Omega_{2}\right)} \frac{\left(\left(n_{2}+1\right)\left(\gamma_{\Omega_{1}}\left(x_{l}\right)+\gamma_{\Omega_{1}}\left(x_{m}\right)\right)+2 n_{2}\right)\left(\gamma_{\Omega_{2}}\left(y_{j}\right)+2\right)}{\gamma_{\Omega_{2}}\left(y_{j}\right)+2 n_{2}+2} \\
& \leq \frac{1}{16} \sum_{e_{i} \in E\left(\Omega_{1}\right)} \sum_{y_{j} \in V\left(\Omega_{2}\right)}\left(\left(\left(n_{2}+1\right)\left(\gamma_{\Omega_{1}}\left(x_{l}\right)+\gamma_{\Omega_{1}}\left(x_{m}\right)\right)+2 n_{2}\right)\left(\gamma_{\Omega_{2}}\left(y_{j}\right)+2\right)\right) \\
& \left(\frac{1}{\gamma_{\Omega_{2}}\left(y_{j}\right)}+\frac{1}{2 n_{2}+2}\right) \\
& =\frac{1}{16} \sum_{e_{i} \in E\left(\Omega_{1}\right)} \sum_{y_{j} \in V\left(\Omega_{2}\right)}\left(\left(n_{2}+1\right)\left(\gamma_{\Omega_{1}}\left(x_{l}\right)+\gamma_{\Omega_{1}}\left(x_{m}\right)\right)+2 n_{2}\right. \\
& \left.+\frac{2\left(\left(n_{2}+1\right)\left(\gamma_{\Omega_{1}}\left(x_{l}\right)+\gamma_{\Omega_{1}}\left(x_{m}\right)\right)+2 n_{2}\right)}{\gamma_{\Omega_{2}}\left(x_{j}\right)}\right) \\
& +\frac{\left(\left(n_{2}+1\right)\left(\gamma_{\Omega_{1}}\left(x_{l}\right)+\gamma_{\Omega_{1}}\left(x_{m}\right)\right)+2 n_{2}\right)\left(\gamma_{\Omega_{2}}\left(y_{j}\right)+2\right)}{2 n_{2}+2} \\
& =\frac{1}{16} \sum_{e_{i} \in E\left(\Omega_{1}\right)}\left(n_{2}\left(n_{2}+1\right)\left(\gamma_{\Omega_{1}}\left(x_{l}\right)+\gamma_{\Omega_{1}}\left(x_{m}\right)\right)+2 n_{2}^{2}\right) \\
& +2\left(\left(n_{2}+1\right)\left(\gamma_{\Omega_{1}}\left(x_{l}\right)+\gamma_{\Omega_{1}}\left(x_{m}\right)+2 n_{2}\right) I D\left(\Omega_{2}\right)\right. \\
& \left.+\left(\left(n_{2}+1\right)\left(\gamma_{\Omega_{1}}\left(y_{l}\right)+\gamma_{\Omega_{1}}\left(x_{m}\right)\right)+2 n_{2}\right)\left(2 m_{2}+2 n_{2}\right)\right) \\
& =\frac{1}{16}\left(n_{2}\left(n_{2}+1\right) M_{1}\left(\Omega_{1}\right)+2 n_{2}^{2} m_{1}+\left(2\left(n_{2}+1\right) M_{1}\left(\Omega_{1}\right)+4 n_{2} m_{1}\right) I D\left(\Omega_{2}\right)\right. \\
& \left.+\left(\left(n_{2}+1\right) M_{1}\left(\Omega_{1}\right)+2 n_{2} m_{1}\right)\left(2 m_{2}+2 n_{2}\right)\right) \text {. }
\end{aligned}
$$

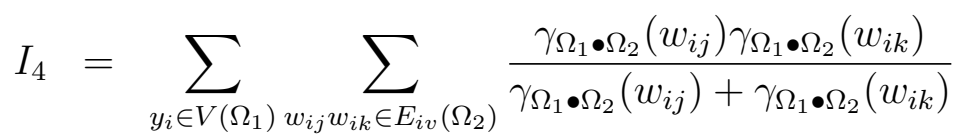

$$
\begin{aligned}
& =\sum_{y_{i} \in V\left(\Omega_{1}\right)} \sum_{w_{j} w_{k} \in E\left(\Omega_{2}\right)} \frac{\left(\gamma_{\Omega_{2}}\left(w_{j}\right)+1\right)\left(\gamma_{\Omega_{2}}\left(w_{k}\right)+1\right)}{\gamma_{\Omega_{2}}\left(w_{j}\right)+\gamma_{\Omega_{2}}\left(w_{k}\right)+2} \\
& \leq \frac{1}{4} \sum_{y_{i} \in V\left(\Omega_{1}\right)} \sum_{w_{j} w_{k} \in E\left(\Omega_{2}\right)}\left(\gamma_{\Omega_{2}}\left(w_{j}\right) \gamma_{\Omega_{2}}\left(w_{k}\right)+\left(\gamma_{\Omega_{2}}\left(w_{j}\right)+\gamma_{\Omega_{2}}\left(w_{k}\right)\right)+1\right) \\
& \left(\frac{1}{\gamma_{\Omega_{2}}\left(w_{j}\right)+\gamma_{\Omega_{2}}\left(w_{k}\right)}+\frac{1}{2}\right)
\end{aligned}
$$

by Jensen's inequality and equality holds if and only if $\gamma_{\Omega_{2}}\left(w_{j}\right)+\gamma_{\Omega_{2}}\left(w_{k}\right)=2$.

$$
=\frac{1}{4}\left(n_{1} I S I\left(\Omega_{2}\right)+\frac{n_{1}}{2} M_{2}\left(\Omega_{2}\right)+n_{1} m_{2}+\frac{n_{1}}{2} M_{1}\left(\Omega_{2}\right)+\frac{n_{1}}{2} H\left(\Omega_{2}\right)+\frac{n_{1} m_{1}}{2}\right) .
$$

$$
\begin{aligned}
I_{5} & =\sum_{x_{i} \in V\left(\Omega_{1}\right)} \sum_{w_{i j} \in V_{i v}\left(\Omega_{2}\right)} \frac{\gamma_{\Omega_{1} \bullet \Omega_{2}}\left(x_{i}\right) \gamma_{\Omega_{1} \bullet \Omega_{2}}\left(w_{i j}\right)}{\gamma_{\Omega_{1} \bullet \Omega_{2}}\left(x_{i}\right)+\gamma_{\Omega_{1} \bullet \Omega_{2}}\left(w_{i j}\right)} \\
= & \sum_{x_{i} \in V\left(\Omega_{1}\right)} \sum_{w_{j} \in V\left(\Omega_{2}\right)} \frac{\left(\left(n_{2}+1\right) \gamma_{\Omega_{1}}\left(x_{i}\right)+n_{2}\right)\left(\gamma_{\Omega_{2}}\left(w_{j}\right)+1\right)}{\left(n_{2}+1\right) \gamma_{\Omega_{1}}\left(x_{i}\right)+n_{2}+\gamma_{\Omega_{2}}\left(w_{j}\right)+1} \\
\leq & \frac{1}{4} \sum_{x_{i} \in V\left(\Omega_{1}\right)} \sum_{w_{j} \in V\left(\Omega_{2}\right)}\left(\left(\left(n_{2}+1\right) \gamma_{\Omega_{1}}\left(x_{i}\right)+n_{2}\right)\left(\gamma_{\Omega_{2}}\left(w_{j}\right)+1\right)\right)\left(\frac{1}{4\left(n_{2}+1\right) \gamma_{\Omega_{1}}\left(x_{i}\right)}+\frac{1}{4\left(n_{2}+1\right)}\right. \\
& \left.+\frac{1}{\gamma_{\Omega_{2}}\left(w_{j}\right)}\right),
\end{aligned}
$$


by Jensen's inequality

$$
\begin{aligned}
= & \frac{1}{4} \sum_{x_{i} \in V\left(\Omega_{1}\right)} \sum_{w_{j} \in V\left(\Omega_{2}\right)}\left(\frac{\gamma_{\Omega_{2}}\left(w_{j}\right)}{4}+\frac{\gamma_{\Omega_{1}}\left(x_{i}\right) \gamma_{\Omega_{2}}\left(w_{j}\right)}{4}+\left(n_{2}+1\right) \gamma_{\Omega_{1}}\left(x_{i}\right)+\frac{\gamma_{\Omega_{1}}\left(v_{i}\right)}{4}+\frac{\left(n_{2}+1\right) \gamma_{\Omega_{1}}\left(x_{i}\right)}{\gamma_{\Omega_{2}}\left(w_{j}\right)}\right. \\
& \left.+\frac{n_{2} d_{\Omega_{2}}\left(w_{j}\right)}{4\left(n_{2}+1\right) \gamma_{\Omega_{1}}\left(x_{i}\right)}+\frac{n_{2} d_{\Omega_{2}}\left(w_{j}\right)}{4\left(n_{2}+1\right)}+n_{2}+\frac{n_{2}}{4\left(n_{2}+1\right) \gamma_{\Omega_{1}}\left(x_{i}\right)}+\frac{n_{2}}{4\left(n_{2}+1\right)}+\frac{n_{2}}{\gamma_{\Omega_{2}}\left(w_{j}\right)}\right) \\
= & \frac{1}{16}\left(2 n_{1} m_{2}+2 n_{2} m_{1}+4 m_{1} m_{2}+8 m_{1} m_{2}\left(n_{2}+1\right)+n_{1} n_{2}+\frac{2 n_{1} n_{2} m_{2}}{n_{2}+1}+4 n_{1} n_{2}^{2}+\frac{n_{1} n_{2}^{2}}{n_{2}+1}\right. \\
& \left.+\frac{\left(2 n_{2} m_{2}+n_{2}^{2}\right) I D\left(\Omega_{1}\right)}{n_{2}+1}+\left(4 n_{2} n_{1}+8 m_{1}\left(n_{2}+1\right)\right) I D\left(\Omega_{2}\right)\right) .
\end{aligned}
$$

Adding $I_{1}$ to $I_{5}$ we arrive the required result.

\section{REFERENCES}

[1] A.T. Balaban, Highly discriminating distance based numerical descriptor, Chem. Phys. Lett. 89 (1982) 399-404.

[2] G.J.Chang, L. Hu, X. Zhu, Circular choromatic number of Mycielski graphs, Discrete Math. 205(1999) 23-37.

[3] J. Devillers, A.T. Balaban, Topological Indices and Related Descriptors in QSAR and QSPR, Gordon and Breach, Amsterdam, 1999.

[4] S. Fajtlowicz, On conjectures on Graffiti-II, Congr. Numer. 60 (1987) 187-197.

[5] D.C. Fisher, P.A. Mckena, E.D. Boyer, Hamiltonicity, diameter, domination, Packing and biclique partitions of the Mycielskis graphs, Discrete Appl. Math. 84(1998) 93-105.

[6] T. Doslic, M. Azari, Falahati-Nezhad, Sharp bounds on the inverse sum indeg invariant, Discrete Appl. Math. 217 (2017) 185-195.

[7] Falahati-Nezhad, M. Azari, The inverse sum indeg invariant of some nanotubes,Studia Ubb Chemia, LXI, 1 (2016) 63-70.

[8] C.K. Gupta, On the symmetric division deg invariant of graph, Southeast Asian Bulletin of Math. 40(2016) $59-80$.

[9] I. Gutman, N. Trinajstić, Graph theory and molecular orbitals. Total $\pi$-electron energy of alternant hydrocarbons, Chem. Phys. Lett. 17 (1972) 535-538.

[10] D.D.F. Liu, Circular choromatic number for iterated Mycielski graphs, Discrete Math. 285(2004) 335-340.

[11] M. Karelson, Molecular Descriptors in QSAR/QSPR, Wiley-Interscience, New York, 2000.

[12] A. Ilić, G. Yu, L. Feng, On the eccentric distance sum of graphs, J. Math. Anal. Appl. 381 (2011) 590-600.

[13] H. Liu, Circular choromatic number and Mycielski construction, J. Graph Theory, 44(2003) 106-115.

[14] J. Mycielski, Sur le colouring des graphes, Colloq. Math. 3 (1955) 161-162.

[15] I. Z. Milovanovć, E. I. Milovanovć, A. Zakić, A short note on graph energy, MATCH Commun. Math. Comput. Chem. 72(2014) 179-182.

[16] Milano Chemometrics \& QSAR research group, molecular descriptors dataset, http://www.moleculardescriptors.eu/dataset/dataset.htm (accessed 18.04.14).

[17] G. Pólya and G. Szego, Problems and Theorems in Analysis, Series, Integral Calculus, Theory of Functions, Springer-Verlag, Berlin, 1972.

[18] K. Pattabiraman, On topological indices of graph transformation, Int. J. Appl. Comput. Math. doi 10.1007/s40819-016-0167-6.

[19] M. Randić, On characterization of molecular branching, J. Am. Chem. Soc. 97 (1975) 6609-6615.

[20] J. Sedlar, D. Stevanović, A. Vasilyev, On the inverse sum indeg inde, Discrete Appl. Math. 184 (2015) 202-212.

[21] V. Sharma, R. Goswami and A.K. Madan, Eccentric connectivity invariant: A novel highly discriminating topological descriptor for structure-property and structure-activity studies, J. Chem. Inf. Comput. Sci. 37 (1997) 273-282. 
[22] B.S. Shetty, V. Lokesha, P.S. Ranjini, On the harmonic invariant of graph operations, Trans. Combin. $4(2015)$ 5-14.

[23] G.H. Shirdel, H. Rezapour and A.M. Sayadi, The hyper-Zagreb index of graph operations, Iranian J. Math. Chem. 4 (2013) 213-220.

[24] R. Todeschini, V. Consonni, Handbook of Molecular Descriptors, Wiley-VCH, Weinheim, 2000.

[25] D. Vukicević. M. Gasperov, Bond aditive mdelling 1. Ariatic indices, Croat. Chem. Acta, 83 (2010) 243-260.

[26] H. Wiener, Structural determination of paraffin boiling points, J. Am. Chem. Soc. 69 (1947) 17-20.

[27] D. Vukicević and A. Graovac, Note on the comparison of the first and second normalized Zagreb eccentricity indices, Acta Chim. Slov. 57 (2010), 524-538.

[28] C. Niculescu, L.E. Persson, Convex functions and their applications: a contemporary approach, Springer, New York 2006. 